\title{
关于亚纯函数的幅角分布"
}

\author{
伍 胜 健 \\ (中国科学院数学研究所, 北京 100080)
}

\section{关锤调 分布、增长、聚值线}

\section{一、引 言}

根据经典的 Weierstrass 和 Hadamard 定理, 一个整函数 $f(z)$ 可以由它的零点的典型 乘积确定到相差一个指数因子 $e^{h(x)}$; 这里 $h(x)$ 为另一整函数. 利用 Nevanlinra 理论, 一 个亚纯函数 $f(z)$ 的零点和极点的分布对 $f(z)$ 也有一定的确定性 ${ }^{[1]}$. 亚纯函数的很多值分 布性质很大程度上可以由它的零点和极点的分布状态来确定. 如果给予零点和极点的分布一 些适当限制, 则可以得到函数的增长性的估计及其规律, 并可以决定亏值的存在性或者得到其 数目的估计. 以往有许多这方面的研究工作 ${ }^{[2-4]}$.

另一方面, 零点和极点的分布也能在某种程度上决定亚纯函数的奇异方向的分布. 例如 关于 Borel 方向分布的著名工作就是基于零点和极点分布而得到的. 以往许多有关奇异方 向(特别是 Julia 方向和 Borel 方向)的问题均可转化为零点和极点的分布问题. 因此研究 亚纯函数的零点和极点的分布是一个很有意义的方向. 本文主要讨论这方面的问题, 具体结 果在以下各节中叙述.

本文采用 Nevanlinna 理论中的通常记号(参考文献 $[1,6]$ ). 除此以外, 我们还需要以下 的定义与记号.

1. 设 $f(z)$ 为平面内的亚纯函数, $k_{1}$ 和 $k_{2}$ 为两个大于 1 的实数 ${ }^{\natural n}$. 我们称 $r \in(0, \infty)$ 关于 $f(z)$ 为 $\left(k_{1}, k_{2}\right)$ 正规 (或简称为 $\left(k_{1}, k_{2}\right)$ 正规), 如果有 $T\left(k_{1}, r, f\right) \leqslant k_{2} T(r, f)$.

2. 设 $0<\beta-\alpha \leqslant 2 \pi, k-\frac{\pi}{\beta-\alpha}, f(z)$ 在 $\mathscr{Q}(\alpha, \beta)-\{\alpha \leqslant \arg z \leqslant \beta\}$ 上亚纯 ${ }^{\text {(8) }}$, 记

$$
\begin{aligned}
& m_{\alpha \beta}(r, f)=\frac{1}{2 \pi} \int_{\alpha}^{\beta} \log +\left|f\left(r e^{i \theta}\right)\right| d \theta ; \\
& A_{\alpha \beta}(r, f)=\frac{k}{\pi} \int_{1}^{r}\left(\frac{1}{t^{k}}-\frac{t^{k}}{r^{2 k}}\right)\left(\log +\left|f\left(t e^{i \alpha}\right)\right|+\log +\left|f\left(t e^{i \beta}\right)\right|\right) \frac{d t}{t} ; \\
& B_{\alpha \beta}(r, f)-\frac{2 k}{\pi r^{k}} \int_{a}^{\beta} \log +\left|f\left(r e^{i \theta}\right)\right| \sin k(\theta-\alpha) d \theta ; \\
& C_{\alpha \beta}(r, f)-2 \sum_{1<b,<r}\left(\frac{1}{\left|b_{\nu}\right|^{k}}-\frac{\left|b_{\nu}\right|^{k}}{r^{2 k}}\right) \sin k\left(\beta_{\nu}-\alpha\right) ;
\end{aligned}
$$

1991-12-11 收稿, 1992-06-10 收修改稿

* 国家自然科学基金资助项目 


$$
S_{\alpha \beta}(r, f)-A_{\alpha \beta}(r, f)+B_{\alpha \beta}(r, f)+C_{\alpha \beta}(r, f),
$$

这里 $b_{v}-\left|b_{v}\right| e^{i \beta_{v}}(\nu-1,2, \cdots)$ 为 $f(z)$ 在 $\bar{Q}(\alpha, \beta)$ 上的极点, 重级极点按重数计算. 如 果每个重数 $>1$ 的极点只计一次, 则相应的 $C_{\alpha \beta}(r, f)$ 记为 $\bar{C}_{\alpha \beta}(r, f)$.

3. 设 $f(z)$ 在平面内超越亚纯. 射线 $\arg z=\theta_{0}$ 称为 $f(z)$ 的一条级 $\geqslant \rho(0 \leqslant \rho \leqslant+\infty)$ 的 $(0, \infty)$ 聚值线, 如果对任意的 $\varepsilon>0$, 有

$$
\varlimsup_{r \rightarrow \infty} \frac{\log \left\{n\left(\Omega\left(\theta_{0}-\varepsilon, \theta_{0}+\varepsilon ; r\right), f-0\right)+n\left(\Omega\left(\theta_{0}-\varepsilon, \theta_{0}+\varepsilon, r\right), f-\infty\right)\right\}}{\log r} \geqslant \rho .
$$

\section{二、亚纯函数的增长性与点和极点的分布}

在这方面我们得到

定理 1 设 $f(z)$ 为开平面内的亚纯函数; $f_{(z)}^{(\prime)}(l \geqslant 0)$ 具有一个有穷非零亏值; $\arg z-\theta_{k}\left(k-1,2, \cdots, m ; 1 \leqslant m<+\infty ; 0 \leqslant \theta_{1}<\theta_{2}<\cdots<\theta_{m}<2 \pi ; \theta_{m+1}-\theta_{1}+2 \pi\right)$ 为 $z$ 平面内 $m$ 条半直线, 并且对任意的 $\varepsilon>0$ 有

$$
\varlimsup_{r \rightarrow \infty} \frac{\log +\bar{n}\left\{\bigcup_{k=1}^{m} \bar{Q}\left(\theta_{k}+\varepsilon, \theta_{k+1}-\varepsilon ; r\right), f-X\right\}}{\log r} \leqslant \rho<+\infty,
$$

则当下级 $\mu<+\infty$ 时, 必有级 $\lambda \leqslant \max \left(\frac{\pi}{\omega}, \rho\right)$, 其中 $\omega-\min _{1<k<m}\left(\theta_{k+1}-\theta_{k}\right)$.

如果在定理 1 中令 $\rho=0$ 和 $l-0$, 这就得到了文献 [4] 中的主要结果, 但他们相应于 (*)式的条件还要更强.

定理 1 的证明需要以下引理:

引理 1 设 $f(z)$ 在开平面亚纯; $a \in \bar{C}$ 使得 $\delta(a, f)>0 ; E-\left\{r, r\right.$ 为 $\left(\dot{k}_{1}, \dot{k}_{2}\right)$ 正规 $\}$, 这里 $k_{1}$ 和 $k_{2}$ 为两个大于 1 的数. 则对每个充分大的 $r \in E$, 使得

$$
\left\{\begin{array}{l}
\log \frac{1}{\left|f\left(r e^{i \phi}\right)-a\right|}>\frac{\delta(a, f)}{4} T(r, f), a \neq \infty ; \\
\log \left|f\left(r e^{i \varphi}\right)\right|>\frac{\delta(a, f)}{4} T(r, f), a-\infty .
\end{array}\right.
$$

成立的 $\varphi(0 \leqslant \varphi<2 \pi)$ 构成的集合 $E(r)$ 有

$$
\operatorname{mes} E(r) \geqslant K \text {, }
$$

这里 $K$ 为一仅依赖 $k_{1}, k_{2}$ 和 $\delta(a, f)$ 而与 $r$ 无关的正数.

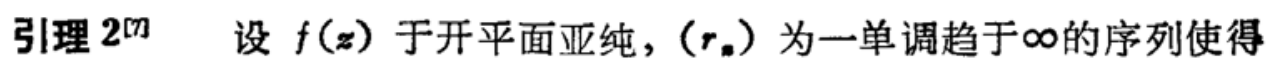

$$
\lim _{n \rightarrow \infty} \frac{\log T\left(r_{n}, f\right)}{\log r_{n}} \leqslant v<+\infty .
$$

再设 $h$ 与 $H(>1)$ 为任意的两个正数, 记 $E-\left\{r, r\right.$ 为 $\left(e^{h}, e^{h H}\right)$ 正规 $\}$, 则

$$
\lim _{x \rightarrow \infty} \frac{1}{\log r_{n}} \int_{E \cap\left[r_{0}, r_{n}\right]} \frac{d t}{t} \geqslant 1-\frac{\nu}{H} \text {. }
$$

引理 $3^{[8]}$ 设 $f(x)$ 在角域 $\bar{Q}(\alpha, \beta)(0<\beta-\alpha \leqslant 2 \pi)$ 上亚纯, $\varepsilon(>0)$ 和 $d(>1)$ 为 任意两个给定的数, 则至多除去一个 $r$ 的集合 $E, \overline{\operatorname{den} s} E<\delta$, 当 $r \in E$ 时有 


$$
m_{\alpha \beta}(r, f) \leqslant K r^{\frac{x}{\beta-\alpha}}\left\{S_{\alpha \beta}(r, f)+1\right\}^{d},
$$

其中 $\boldsymbol{K}$ 为与 $\boldsymbol{r}$ 无关的常数.

引理 $4^{[9]}$ 设 $f(z)$ 为超越亚纯函数和 $M>1$, 则可能存在一个 $r$ 的集合 $E(M)$, $\overline{\log \operatorname{den} s} E(M) \leqslant\left(2 e^{M-1}-1\right)^{-1}$, 使得对任意的正整数 $l$ 有

$$
\varlimsup_{\substack{r \\ r \in B(M)}} \frac{T(r, f)}{T\left(r, f^{(l)}\right)} \leqslant 3 e M .
$$

\section{三、亚纯函数的 $(0, \infty)$ 聚值线的分布}

以下是我们得到的主要结果.

定理 2 设 $f(z)$ 在开平面亚纯, 其级为 $\lambda(0<\lambda<+\infty)$, 下级为 $\mu, \rho$ 为一正数适 合条件 $\mu \leqslant \rho \leqslant \lambda, a_{i}(i=1,2, \cdots, p ; 1 \leqslant p<+\infty)$ 为一组互相判别的有穷非零复数使得 $\delta\left(a_{i}, f\right)>0$. 则在角顶位于原点, 开度大于

$$
\max \left\{\frac{\pi}{\rho}, 2 \pi-\frac{4}{\rho} \sum_{i=1}^{\infty} \arcsin \sqrt{\frac{\delta\left(a_{i}, f\right)}{2}}\right\}
$$

的任意角域 $Q$ 内, $f(z)$ 至少具有一条级 $\geqslant \rho$ 的 $(0, \infty)$ 聚值线.

定理 3 设亚纯函数 $f(z)$ 的级为 $\lambda(0<\lambda<+\infty)$, 下级为 $\mu, \rho$ 为一数适合条件 $\boldsymbol{\mu} \leqslant p \leqslant \lambda$. 再设 $f(z)$ 具有 $m(1 \leqslant m<+\infty)$ 条级 $\geqslant \rho$ 的 $(0, \infty)$ 聚值线 $\arg z-\theta_{k}(0 \leqslant$ $\left.\theta_{1}<\theta_{2}<\cdots<\theta_{m} ; \theta_{m+1}-\theta_{1}+2 \pi\right)$. 如果 $f(x)$ 具有一个有穷非零亏值, 则有 $\lambda \leqslant \frac{\pi}{\omega}$, 这里 $\dot{\omega}-\min _{1<k<m}\left(\theta_{k+1}-\theta_{k}\right)$.

定理 4 设亚纯函数 $f(z)$ 的级为 $\lambda(0<\lambda<+\infty), \rho$ 为一正数适合条件 $\rho \leqslant \lambda$. 再 设角域 $Q(\alpha, \beta)$ 的开度大于 $\frac{\pi}{\rho}$. 如果 $f^{\prime}(\boldsymbol{s})$ 在 $Q(\alpha, \beta)$ 内具有一条级 $\geqslant \rho$ 的 $(0, \infty)$ 聚值 线,则 $f(z)$ 在 $Q(\alpha, \beta)$ 内也至少具有一条级 $\geqslant \rho$ 的 $(0, \infty)$ 聚值线.

从定理 2 和 4 容易推出文献 [10]中的主要结果, 从定理 3 和 4 容易推出文献 [11]中的主 要结果.

以上定理的证明主要是基于以下的引理:

引理 5 设 $f(z), \lambda, \mu$ 和 $\rho$ 满足定理 2 的条件, $\left(r_{n}\right)$ 为一列 $\rho$ 级 Pólya 峰序列. 再 设 $f(\boldsymbol{z})$ 在 $\boldsymbol{Q}\left(\theta_{1}, \theta_{2}\right)\left(0<\theta_{2}-\theta_{1} \leqslant 2 \pi\right)$ 内不具有级 $\geqslant \rho$ 的 $(0, \infty)$ 聚值线. 若存在一个有 穷非零复数 $a_{0}$, 使得对每个充分小的正数 $\varepsilon$, 当 $n$ 充分大时有

$$
\operatorname{mes} E\left(\theta ; \theta_{1}<\theta<\theta_{2}, \log \frac{1}{\left|f\left(r_{n} e^{i \theta}\right)-a_{0}\right|}>r_{n}^{\rho-s}\right)>K,
$$

其中 $K$ 为不依赖 $\varepsilon$ 与 $n$ 的正数, 则有 $\theta_{2}-\theta_{1} \leqslant \frac{\pi}{\rho}$.

致谢本文是在杨乐教授指导下完成的, 本文初稿还得到他的仔细审阅,作者向他表示 最深切的谢意. 


\section{参考文献}

[1] Hayman, W. K., Meromorphic Functions, Oxford, 1964, 21.

[2] Edrei, A., Fuchs, W. H. J., Trans. Amer. Math. Soc., 93(1959), 292-328.

[3] 杨乐、张广厚, 中国科学, 1981, 12: 1429-1439.

[4] 张广厚、伍鹏程, 中国科学, A 辑, 1985, 5: 423-434.

[5] 杨乐、张广厚, Sci. Sinica, 19(1976), 445-459.

[6] 杨乐,值分布论及其新研究, 科学出版社, 1982 .

[7] Hayman, W. K., Proc. London Math. Soc., 24(1972), 590-624.

[8] Goldberg, A. A., Ostrovskii, I. V., The Distribution of Values of Meromorphic Functions (in Russian), Izdat. Nauk, Moscow, 1970.

[9] Hayman, W. K., Miles, J., Complex Variables, 12(1989), 245-260.

[10] 杨乐,中国科学,数学专辑(1), 1979, 149-164.

[11] 杨乐、张广厚, Sci. Sinica, 16(1973), 465-482. 